\title{
Terahertz Resonant Detection by Plasma Waves in Nanometric Transistors
}

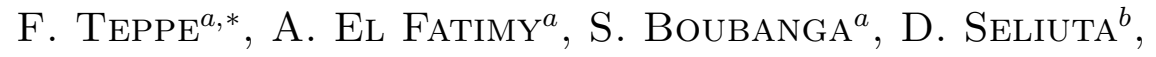 \\ G. VAlusis ${ }^{b}$, B. Chenaud ${ }^{c}$ And W. KnaP ${ }^{a}$ \\ ${ }^{a}$ GES, CNRS-Universite Montpellier 2, 34095 Montpellier, France
}

${ }^{b}$ Semiconductor Physics Institute, A. Goštauto 11, LT- 01108 Vilnius, Lithuania

${ }^{c}$ University of Tokyo, Komaba 3-8-1, Meguro-ku, Tokyo, 153-8902, Japan

The plasma waves in gated two-dimensional electron gas have a linear dispersion law, similar to the sound waves. The transistor channel is acting as a resonator cavity for the plasma waves, which can reach frequencies in the $\mathrm{THz}$ range for a sufficiently short gate length field effect transistors. A variety of possible applications of field effect transistor operating as a $\mathrm{THz}$ device were suggested. In particular, it was shown that the nonlinear properties of plasma oscillations can be utilized for $\mathrm{THz}$ tunable detectors. During the last few years $\mathrm{THz}$ detection related to plasma wave instabilities in nanometer size field effect transistors was demonstrated experimentally. In this work we review our recent experimental results on the resonant plasma wave detection at cryogenic and room temperatures.

PACS numbers: 71.20.Nr, 71.45.Lr, 72.30.+q

\section{Introduction}

The terahertz $(\mathrm{THz})$ range of frequencies is often referred to as the "terahertz gap", since it lies in between the frequency ranges of electronic and photonic devices and it is hardly achieved from the both sides. Therefore, the development of the THz emitters and detectors is of the high importance. Dyakonov and Shur $[1,2]$ proposed to use the nonlinear properties of plasma excitations in $2 \mathrm{D}$ gated electron gas for terahertz detectors, mixers [3, 4], and $\mathrm{THz}$ radiation sources [5]. The plasma waves in a field effect transistor (FET) have a linear dispersion law [1], $\omega=s k$ where $s=\sqrt{e\left(V_{\mathrm{g}}-V_{\mathrm{th}}\right) / m}$ is the plasma wave velocity, $V_{\mathrm{g}}$ is the gate voltage, $V_{\text {th }}$ is the threshold voltage, $e$ is the electronic charge and $m$ is the electron effective mass. This plasma wave velocity is typically much larger than the electron drift velocity. A short FET channel of a given gate length, $L_{\mathrm{g}}$, acts as a resonant

*corresponding author; e-mail: teppe@ges.univ-montp2.fr 
"cavity" for these waves with the eigenfrequencies given by $\omega_{N}=\omega_{0(1+2 N)}$, where $N=1,2,3 \ldots$ The fundamental plasma frequency is

$$
\omega_{0}=\frac{\pi}{2 L_{\mathrm{g}}} \sqrt{e \frac{V_{\mathrm{g}}-V_{\mathrm{th}}}{m}},
$$

when $\omega_{0} \tau \ll 1$, where $\tau$ is the momentum relaxation time, the detector response is a smooth function of $\omega$ and $V_{\mathrm{g}}$ (broad band detector) [6]. When $\omega_{0} \tau \gg 1$, the FET can operate as a resonant detector. For the submicron gate lengths, the resonant detection frequency $f=\omega_{0} / 2 \pi$ can reach the $\mathrm{THz}$ range [1]. In that case the electron flow in the channel may become unstable (at certain boundary conditions) with respect to formation of the resonant plasma oscillations.

In this paper we present the detection of $\mathrm{THz}$ radiation by high electron mobility nanometer InGaAs/AlInAs transistors at low temperature. The photovoltaic type of response was observed at the $1.8-3.1 \mathrm{THz}$ frequency range, which is far above the cut-off frequency of the transistors [7]. The resonant response was observed and was found to be tunable by the gate voltage. We also present our experimental results on room temperature tunable THz detection by InGaAs/AlInAs nanotransistors [8]. Indeed, we have demonstrated that the detection of $\mathrm{THz}$ radiations by plasma waves might be strongly modified by the application of a dc drain current. We found that driving a transistor into the saturation region enhances the non-resonant detection and can lead to the resonant detection [9-11].

\section{Results and discussion}

In this first part we present the detection of sub-terahertz and $\mathrm{THz}$ radiation by $60 \mathrm{~nm}$ gate length InGaAs/AlInAs transistors in the 1.8-3.1 $\mathrm{THz}$ frequency range [7]. The experiments were performed in the temperature range from 10 to $80 \mathrm{~K}$ with a closed-cycle helium cryostat. The response to the terahertz radiation was measured as a dc voltage on the open drain as a function of gate voltage. The source was grounded.

The results of the photoresponse in the InGaAs/InAlAs transistor exposed to the radiation of $2.5 \mathrm{THz}$ frequency as function of the gate voltage measured at various temperatures are shown in Fig. 1a. Above $100 \mathrm{~K}$ only non-resonant detection is observed as a broad band peak. With the decrease in temperature, below $80 \mathrm{~K}$, the additional peak appears as a shoulder on the temperature-independent background of the non-resonant detection. We attribute this behavior to the resonant detection of $\mathrm{THz}$ radiation by plasma waves. To support this assumption, we have measured the response at different excitation frequencies of 1.8, 2.5, and $3.1 \mathrm{THz}$ at $10 \mathrm{~K}$. The experimental results are displayed in Fig. 1b. For comparison, we have plotted the estimate of the plasma frequency as a function of gate voltage obtained using Eq. (1.1), shown as a continuous line in Fig. 1c. One can see that with the increase in excitation frequency from 1.8 to $3.1 \mathrm{THz}$ the plasmon resonance moves with the gate voltage, in rough agreement with Eq. (1.1). Nevertheless, the width of the observed resonant peaks was measured to be one decade 
broader than expected by the theory. This experimental result shows that additional mechanisms of plasma wave damping must be involved. These mechanisms might include effect related to ballistic transport [12], or oblique plasmons on the gated part of the channel, and/or leaking of the gated plasmons in the ungated part of the channel.

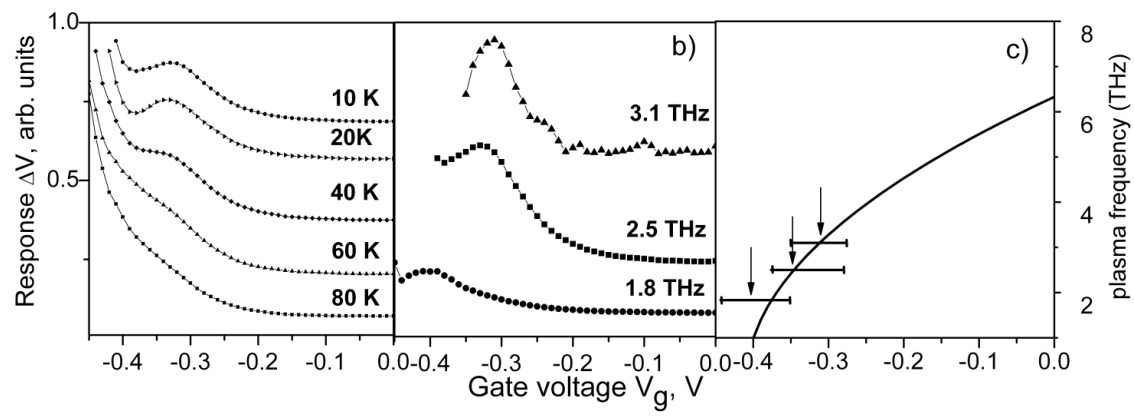

Fig. 1. (a) Response of InGaAs/InAlAs device exposed to the terahertz radiation as a function of the gate voltage at different temperatures $(80 \mathrm{~K}$ down to $10 \mathrm{~K})$. Excitation frequency is $2.5 \mathrm{THz}$. (b) Response as a function of the gate voltage at different (1.8, 2.5 , and $3.1 \mathrm{THz}$ ) frequencies, $T=10 \mathrm{~K}$. c) Position (indicated by arrows) of resonance maxima as a function of gate voltage. The calculated plasmon frequency as a function of the gate voltage using Eq. (1.1) for $V_{\mathrm{th}}=-0.41 \mathrm{~V}$ is shown by the solid line. The error bars correspond to the line width of the measured plasmon resonance peaks.

In this second part we present the results on the plasma wave related $\mathrm{THz}$ detection at room temperature. Recently it has been predicted theoretically that applying a drain-to-source current [9] can lead to room temperature resonant detection of $\mathrm{THz}$ radiations. We have demonstrated experimentally that driving a transistor into the saturation region enhances the non-resonant detection and can lead to the resonant detection $[8,10,11]$ even if the condition $\omega_{0} \tau \gg 1$ is not satisfied. The physical reason is that the effective decay rate for plasma oscillations in the condition of hot drifting electrons becomes equal to $1 / \tau_{\text {eff }}=1 / \tau-2 v / L_{\mathrm{g}}$, where $v$ is the electron drift velocity and $L_{\mathrm{g}}$ the gate length, and becomes longer when applying current. As $\omega \tau_{\text {eff }}$ becomes on the order of unity, the detection becomes resonant.

The experiments were performed on a $50 \mathrm{~nm}$ gate length InGaAs/InAlAs high electron mobility transistor (HEMT). The photoresponse measurements were performed with backward wave oscillator (BWO) source. The photoconductive response versus gate voltage for frequencies from $473 \mathrm{GHz}$ up to $679 \mathrm{GHz}$ is shown in Fig. 2 while keeping constant the source drain voltage of $0.3 \mathrm{~V}$. One can see for the lowest frequency that only typical non-resonant signal is observed. For higher frequencies, after a typical increase in the non-resonant background signal with applied drain current, the resonant structure starts to grow. 


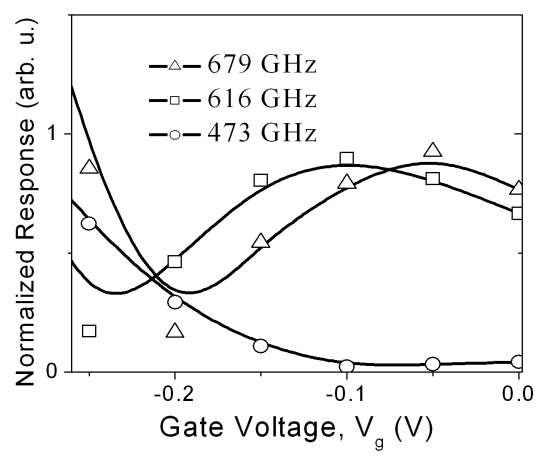

Fig. 2. Photo-induced drain-source voltage as a function of gate bias for different external frequencies $(679 \mathrm{GHz}, 616 \mathrm{GHz}$, and $473 \mathrm{GHz})$ at a fixed value of applied drain-source voltage of $0.3 \mathrm{~V} . T=300 \mathrm{~K}$ (After Ref. [8]).

The main experimental result shown in this part of the letter is that the position of the resonance line shifts with external frequency. Figure 3 reports the experimentally observed variation of the maxima of the gate voltage for three different fixed drain-to-source voltages $(0.1 \mathrm{~V}$ - triangles; $0.2 \mathrm{~V}$ - circles; $0.3 \mathrm{~V}$ - squares). One can see that by increasing the external frequency of BWO the resonance shifts to higher gate voltage for different values of fixed source-drain voltage.

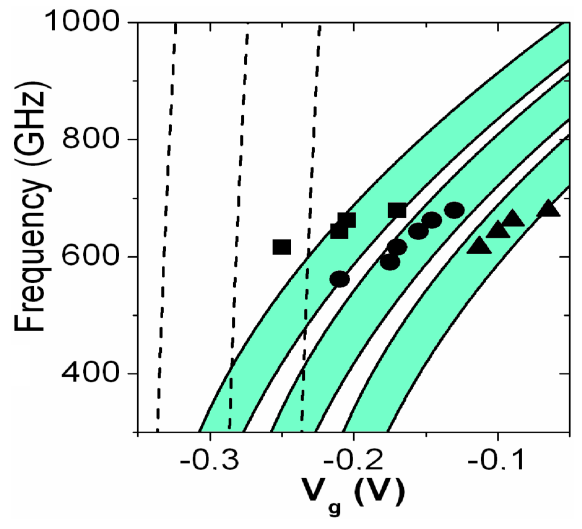

Fig. 3. Maxima of resonant detection corresponding to different frequencies of the radiation source at three different fixed drain-to-source voltages. Dashed lines, respectively, from left to right are calculations using Eq. (2.1) for three values of the effective threshold voltage $V_{\mathrm{th}}=-0.35,-0.3$, and $-0.25 \mathrm{~V}$, corresponding to three values of applied $V_{\mathrm{d}}=0.1,0.2$, and $0.3 \mathrm{~V}$. Solid lines are calculations using Eq. (2.2) at two different values of electron drift velocity. Gray areas represent drift velocity range (between $2 \times 10^{5}$ and $3.5 \times 10^{5} \mathrm{~m} / \mathrm{s}$ ) (After Ref. [8]). 
According to plasma wave detection theory taking into account the drainto-source current, the frequency of plasma wave oscillations $f$ depends on the gate length $L_{\mathrm{g}}$, the plasma wave velocity $s=\sqrt{e\left(V_{\mathrm{g}}-V_{\mathrm{th}}\right) / m}$ and the drift velocity $v$ as [9-11]:

$$
f=\frac{s}{4 L_{g}}\left(1-\frac{v^{2}}{s^{2}}\right) .
$$

We have here to introduce an effective threshold voltage $V_{\mathrm{th}}^{\prime} \approx V_{\mathrm{th}}+V_{\mathrm{d}} / 2$, where $V_{\text {th }}=-0.4 \mathrm{~V}$, to describe resonant peak shifting with applied source-drain current/voltage. The total source-drain voltage used was $V_{\mathrm{d}}=0.1 \mathrm{~V}, 0.2 \mathrm{~V}$, and $0.3 \mathrm{~V}$, and the effective threshold voltage was $-0.35 \mathrm{~V},-0.3 \mathrm{~V}$, and $-0.25 \mathrm{~V}$, respectively.

Dashed lines in Fig. 3 are calculations using Eq. (2.1) taking $V_{\mathrm{g}}-V_{\mathrm{th}^{\prime}}$ as fitting parameter to describe resonant peak shifting with applied $V_{\mathrm{d}}$. The electron drift velocity in the saturation regime is assumed to be $2 \times 10^{5} \mathrm{~m} / \mathrm{s}$. One can see that the calculation does not reproduce well the increase in the gate voltage with frequency. The quantitative interpretation based on these equations allows to get only approximate description of experimental data. In fact, the transistor channel region under the gate cannot be considered as separated from the other parts of the transistor. The transistor consists not only of the gated part but also of the ungated part that is covered by the cap layer. As predicted in [13], the cap regions significantly affect the plasma oscillation spectrum in HEMTs: the resonant plasma frequencies dramatically decrease with increasing cap region length. In order to take into account the effect of such cap layers, a correction to Eq. (2.1) gives

$$
f \approx \frac{s}{L_{\mathrm{g}}+2 L_{\mathrm{c}}}\left(1-\frac{v^{2}}{s^{2}}\right),
$$

where $L_{\mathrm{c}}$ is the cap layer length $\left(L_{\mathrm{c}}=500 \mathrm{~nm}\right)$.

Solid lines in Fig. 3 are calculations using Eq. (2.2) at two different values of electron drift velocity $\left(2 \times 10^{5}\right.$ and $\left.3.5 \times 10^{5} \mathrm{~m} / \mathrm{s}\right)$ for each effective threshold voltage used before (i.e. $V_{\mathrm{th}^{\prime}}=-0.35 \mathrm{~V},-0.3 \mathrm{~V}$, and $-0.25 \mathrm{~V}$ ). Filled areas represent drift velocity range which can match the observed frequency dependence as a function of gate voltage. The calculated increases in frequency with gate voltage can now be qualitatively explained. However, we do not understand why cap regions affect the plasma waves only when a current is flowing through the channel. A full quantitative interpretation of our results requires a more complete theoretical development.

\section{Conclusion}

In conclusion, we have experimentally shown that the resonant detection of $\mathrm{THz}$ electromagnetic waves in InGaAs nanometric HEMTs can be tuneable with gate voltage at cryogenic temperatures. While varying the temperature in the range from 10 to $80 \mathrm{~K}$ and the excitation frequency within 1.8-3.1 THz, we have shown that the detection is due to excitation of resonant plasma waves in the 
transistor channel. We have also shown that the $\mathrm{THz}$ detection by plasma waves can be resonant and voltage tuneable even at room temperature by applying a drain-to-source voltage in InGaAs nanometric HEMT's channel. We have also shown that, in these conditions, the cap layer regions significantly affect the plasma oscillation spectrum in HEMTs.

\section{Acknowledgments}

The work was supported by CNRS and GDR-E project "Semiconductor sources and detectors of $\mathrm{THz}$ frequencies". We acknowledge the region of Languedoc-Roussillon through the "Terahertz Platform" project, the French Ministry of Finance and Industry through "Nano2008" grant and the European Commission with "PullNano" project. Financial support from the joint FrenchLithuanian research programme Gilibert/Egide is also gratefully acknowledged.

\section{References}

[1] M. Dyakonov, M.S. Shur, Phys. Rev. Lett. 71, 2465 (1993).

[2] M.I. Dyakonov, M.S. Shur, IEEE Trans. Electron Dev. 43, 380 (1996).

[3] T. Otsuji, M. Hanabe, O. Ogawara, Appl. Phys. Lett. 85, 2119 (2004).

[4] J. Torres, P. Nouvel, A. Akwoue-Ondo, L. Chusseau, F. Teppe, A. Shchepetov, S. Bollaert, Appl. Phys. Lett. 89, 201101 (2004).

[5] N. Dyakonova, F. Teppe, J. Łusakowski, W. Knap, M. Levinshtein, S. Bollaert, A. Cappy, J. Appl. Phys. 97, 114313 (2005).

[6] W. Knap, F. Teppe, Y. Meziani, N. Dyakonova, J. Lusakowski, F. Boeuf, T. Skotnicki, D. Maude, S. Rumyantsev, M.S. Shur, Appl. Phys. Lett. 85, 675 (2004).

[7] A. El Fatimy, F. Teppe, N. Dyakonova, W. Knap, D. Seliuta, G. Valusis, A. Shchepetov, Y. Roelens, S. Bollaert, A. Cappy, S. Rumyantsev, Appl. Phys. Lett. 89, 131926 (2006); A. El Fatimy, S.B. Tombet, F. Teppe, W. Knap, D.B. Veksler, S. Rumyantsev, M.S. Shur, N. Pala, R. Gaska, Q. Fareed, X. Hu, D. Seliuta, G. Valusis, C. Gaquiere, D. Theron, A. Cappy, Electron. Lett. 42, 1342 (2006).

[8] F. Teppe, M. Orlov, A. El Fatimy, A. Tiberj, W. Knap, J. Torres, V. Gavrilenko, A. Shchepetov, Y. Roelens, S. Bollaert, Appl. Phys. Lett. 89, 222109 (2006).

[9] D. Veksler, F. Teppe, A.P. Dmitriev, V.Yu. Kachorovskii, W. Knap, M.S. Shur, Phys. Rev. B 73, 125328 (2006).

[10] F. Teppe, D. Veksler, V. Yu. Kachorovski, A.P. Dmitriev, X. Xie, X.-C. Zhang, S. Rumyantsev, W. Knap, M.S. Shur, Appl. Phys. Lett. 87, 022102 (2005).

[11] F. Teppe, W. Knap, D. Veksler, V.Yu. Kachorovskii, A.P. Dmitriev, S. Rumyantsev, M.S. Shur, Appl. Phys. Lett. 87, 052107 (2005).

[12] J. Lusakowski, W. Knap, Y. Meziani, J.-P. Cesso, A. El Fatimy, R. Tauk, N. Dyakonova, G. Ghibaudo, F. Boeuf, T. Skotnicki, Appl. Phys. Lett. 87, 053507 (2005).

[13] V. Ryzhii, A. Satou, W. Knap, M.S. Shur, J. Appl. Phys. 99, 084507 (2006). 